\title{
ENHANCEMENT OF COMBUSTION BY DRIFT IN A COUPLED REACTION-DIFFUSION MODEL*
}

\author{
LAM RAGA A. MARKELY ${ }^{\dagger}$, DAVID ANDRZEJEWSKI ${ }^{\ddagger}$, ERICK BUTZLAFF ${ }^{\S}$, AND \\ ALEXANDER KISELEV $₫$
}

\begin{abstract}
We study analytically and numerically a model describing front propagation of a KPP reaction in a fluid flow. The model consists of coupled one-dimensional reaction-diffusion equations with different drift coefficients. The main rigorous results give lower bounds for the speed of propagation that are linear in the drift coefficient, which agrees very well with the numerical observations. In addition, we find the optimal constant in a functional inequality of independent interest used in the proof.
\end{abstract}

Key words. Reaction-diffusion, combustion, fluid flow.

1991 MSC Subject Classifications. 35K57, 35K15, 35B40

\section{Introduction}

Many reaction processes in nature and engineering take place in a moving fluid. The creation of ozone in the atmosphere, the nuclear explosion of a supernova, pattern formation in morphogenesis, wild fires, and the gasoline transformation in an internal combustion engine are just a few examples. The effect of fluid flow on a reaction process can be profound, especially if the flow is strong. The problem has been studied for many years by engineers, physicists, and mathematicians alike. One of the most extensively studied mathematical models of the premixed reaction process is the reaction-diffusion equation and systems. The advection-reaction-diffusion equation is given by

$$
T_{t}+A u \cdot \nabla T=\kappa \Delta T+M f(T) .
$$

Here $u(x)$ is the fluid flow, which we will assume is time-independent and passive. The coefficient $A$ is the flow strength parameter, $\kappa$ is the diffusivity, $M$ is the reaction strength, and $f(T)$ is the reaction term. The function $T(x, t)$ is normalized so that $0 \leq T(x, t) \leq 1$, and can stand for normalized temperature, the mass fraction of a reactant, or a share of a population with a certain feature, depending on the problem. Classical works of Fisher and Kolmogorov-Petrovski-Piskunov (KPP) [12, 21] first considered equation (1.1) in the dimension $d=1$ and with $u=0$. These works modeled the propagation of an advantageous gene in the population, and established existence and, in a certain sense, stability of the traveling fronts. The extensions have occupied some of the best efforts of mathematicians since. Equation (1.1) in several spacial dimensions in the absence of advection $(u=0)$ is by now fairly well understood: the existence of traveling waves, stability, and asymptotic propagation properties have

\footnotetext{
${ }^{*}$ Received: September 29, 2005; accepted (in revised version): January 27, 2006. Communicated by Jack Xin.

${ }^{\dagger}$ Department of Chemical and Biological Engineering, University of Wisconsin, Madison, WI 53706, USA

${ }^{\ddagger}$ Department of Mathematics, University of Wisconsin, Madison, WI 53706, USA.

$\S$ Department of Mathematics, University of Wisconsin, Madison, WI 53706, USA.

『Department of Mathematics, University of Wisconsin, Madison, WI 53706, USA (kiselev@ math.wisc.edu).

This work has been a part of Collaborative Undergraduate Research Lab at the University of Wisconsin. Support of the NSF VIGRE grant is acknowledged.
} 
been extensively studied (see, e.g. $[3,13,14,31]$ where many more references can be found). The effects of advection, however, are very important in many situations in combustion and more general chemical reactions [29, 32, 34] as well as in biology and ecology $[22,24]$.

One important phenomena that is observed in many situations is the enhancement of the reaction rate by fluid motion. The physical reason for this observed speed-up is believed to be that fluid advection tends to increase the area available for reaction. Recently, there has been significant progress in the mathematical understanding of the reaction rate enhancement for several different classes of flows $[1,2,4,9,10,15$, $19,18,16,17,20,23,25,26,27,30]$. The papers $[5,33]$ provide excellent reviews of some of these results and further references.

In this paper, our goal is to study reaction enhancement within a framework of a model given by a coupled system of one-dimensional advection-reaction-diffusion equations:

$$
\left(T_{j}\right)_{t}+A_{j}\left(T_{j}\right)_{x}-\left(T_{j}\right)_{x x}=T_{j}\left(1-T_{j}\right)+\alpha\left(T_{j-1}-T_{j}\right)+\alpha\left(T_{j+1}-T_{j}\right),
$$

$j=1, \ldots, N$. We assume that the model is periodic in $j$; that is, in the equation for $T_{1}(x)$, we replace $T_{0}(x)$ with $T_{N}(x)$, and in the equation for $T_{N}(x)$, we put $T_{1}(x)$ instead of $T_{N+1}(x)$. The initial data always satisfies $0 \leq T_{j}(x, 0) \leq 1$, and it is a simple consequence of the maximum principle that $T_{j}(x, t)$ remains within these bounds for all times (see Section 2). Our reaction term is the classical KPP [21], and for simplicity we set $\kappa=M=1$ (these parameters are normalized by a simple rescaling of time and space). We assume that $\sum_{j=1}^{N} A_{j}=0$, which corresponds to the mean zero flow. Any nonzero mean is taken into account by switching to a moving system of coordinates; we are interested in a non trivial effect of front stretching rather than simple transfer with a constant speed. One can regard (1.2) as a model for reaction in a layered fluid, where different layers move with different speeds. The role of the parameter $\alpha>0$ is to provide diffusive coupling between different layers, and its meaning will be further discussed in Section 4. Our main interest is in the case of the large coefficients $A_{j}$, where the influence of the drift is most pronounced. We consider the front-like initial data where $T_{j}(x, 0) \rightarrow 0$ as $x \rightarrow \infty$ and $T_{j}(x, 0) \rightarrow 1$ as $x \rightarrow-\infty$ exponentially fast (we will state the exact conditions in Section 2). We adopt the following natural quantity (called the bulk burning rate in [9]) as the main measure of the reaction rate:

$$
V(t)=\frac{1}{N} \sum_{j=1}^{N} \int_{-\infty}^{\infty}\left(T_{j}\right)_{t}(x) d x .
$$

One of the results that we prove is as follows.

THEOREM 1.1. Assume that the initial data $T_{j}(x, 0)$ are front like $(2.2)$. Then there exists a universal constant $C$ such that for any $\tau>0$, we have

$$
\frac{1}{\tau} \int_{0}^{\tau} V(t) d t \geq \frac{C}{N} \sum_{j=1}^{N}\left|A_{j-1}-A_{j}\right|\left[\frac{1}{\alpha \tau^{2}}+\frac{1}{\alpha \tau}+\frac{1}{\tau}+\alpha+1\right]^{-1} .
$$

There are several noteworthy properties the lower bound (1.4) possesses. Firstly, the bound is linear in the flow strength. Secondly, the estimate clearly shows the need to wait for a certain time $\tau$ before the front propagation (and thus the reaction rate) 
stabilizes and the long-time lower bound is achieved. If the coupling constant $\alpha$ is large, we have the lower bound $C A \alpha^{-1}$ starting from times $\tau \approx 1$. If $\alpha$ is small, the lower bound is $C A$, starting from times $\tau \approx \alpha^{-1}$. We interpret these results further in Sections 4 and 5. The numerical experiments discussed in Section 5 show very good qualitative agreement with (1.4).

We remark that the result of Theorem 1.1 is reminiscent of the bounds proved in [9] for the two dimensional equation (1.1) with a shear flow $u=(u(y), 0)$. This is not surprising since our system (1.2) can be regarded as a discrete model of a shear flow. However, the proofs in the case of model (1.2) are conceptually much more transparent, the estimates are more precise, and the dependence on the averaging time is more explicit.

The paper is organized as follows. In Section 2 we collect some background estimates which are needed in the proof of main results. In particular, we prove a sharp version of a functional inequality which was first discovered in [9]. The inequality is somewhat reminiscent of the indeterminacy principle of quantum mechanics. Although finding a sharp constant is not particularly important for the key results of this paper, we feel that the result is elegant (even if elementary) and may be of independent interest. In Section 3 we discuss a simpler two-layer case. In Section 4 we prove Theorem 1.1, and some related results for a slightly more general model. In Section 5 we describe the numerical simulations.

\section{The auxiliary tools and the optimal constant}

We make our model slightly more general by associating a width $h_{j}$ with each layer. By dimensionality of the discrete gradient terms providing coupling of the neighboring layers in (1.2), the parameter $\alpha$ is made to depend on $j$, with $\alpha_{j}=\kappa / h_{j}$ :

$$
\left(T_{j}\right)_{t}+A_{j}\left(T_{j}\right)_{x}-\left(T_{j}\right)_{x x}=T_{j}\left(1-T_{j}\right)+\alpha_{j}\left(T_{j-1}-T_{j}\right)+\alpha_{j}\left(T_{j+1}-T_{j}\right) .
$$

The parameter $\kappa$ now plays the role of diffusivity between the layers. As opposed to the diffusivity in $x$ direction, this diffusion coefficient cannot be set to one by a simple rescaling. Our mean zero flow condition now reads $\sum_{j=1}^{N} h_{j} A_{j}=0$. Set $H=\sum_{j=1}^{N} h_{j}$.

We first prove a lemma on the preservation of spacial decay of the solutions of (2.1) that will allow us to manipulate the equations, in particular to integrate by parts.

Lemma 2.1. Assume that $T_{j}(x, t), j=1, \ldots, N$ satisfy (2.1) and that the initial data $T_{j}(x, 0)$ satisfy

$$
T_{j}(x, 0)<C_{0} e^{-\lambda x}, 1-T_{j}(x, 0)<C_{0} e^{\lambda x},\left|\left(T_{j}(x, 0)\right)_{x}\right|<C_{0} e^{-\lambda|x|}
$$

for all $j$ and some $\lambda>0$. Assume that $c_{1}$ is such that

$$
c_{1} \geq \max _{j}\left|A_{j}\right|+\lambda+\lambda^{-1}\left(1+2 \max _{j} \alpha_{j}\right) .
$$

Then for all $x, t>0$ we have

$$
T_{j}(x, t) \leq C_{0} e^{-\lambda\left(x-c_{1} t\right)}, 1-T_{j}(x, t)<C_{0} e^{\lambda\left(x+c_{1} t\right)},\left|\left(T_{j}(x, t)\right)_{x}\right|<C_{0} e^{\lambda\left(|x|+c_{1} t\right)} .
$$

Proof. Let us start by establishing the first bound in (2.4); the second bound can be proved by an identical argument. Set $\phi(x, t)=e^{-\lambda\left(x-c_{1} t\right)}$, and $G_{j}(x, t)=C_{0} \phi(x, t)-$ $T_{j}(x, t)$. A direct computation using (2.3) shows that

$$
\phi_{t}+\max _{\mathrm{j}}\left|\mathrm{A}_{\mathrm{j}}\right| \phi_{\mathrm{x}}-\phi_{\mathrm{xx}}-\left(1+2 \max _{\mathrm{j}} \alpha_{\mathrm{j}}\right) \phi \geq 0
$$


for any $j$. Note that $G_{j}(x, 0)>0$ in view of $(2.2)$. On the contrary, assume that the first bound in (2.4) is not satisfied and that $t_{0}>0$ is the first time such that there exists $j$ and $x_{0}$ so that $G_{j}\left(x_{0}, t_{0}\right)=0$. Combining (2.5) with (2.1), we find that

$$
\left(G_{j}\right)_{t}+A_{j}\left(G_{j}\right)_{x}-\left(G_{j}\right)_{x x} \geq 0
$$

for all $x, t \leq t_{0}$. Now, in $(2.6), G_{j}(x, 0)>0$ for all $x$ and $G_{j}\left(x_{0}, t_{0}\right)=0$ contradict the well-known maximum principle for parabolic PDE (see e.g. [11]). The proof of the second inequality in (2.4) is similar and is omitted. To prove the bound for $\left(T_{j}\right)_{x}$, differentiate $(2.1)$; denoting $H_{j}=\left(T_{j}\right)_{x}$, we have

$$
\left(H_{j}\right)_{t}+A_{j}\left(H_{j}\right)_{x}-\left(H_{j}\right)_{x x}=H_{j}\left(1-2 T_{j}\right)+\alpha_{j}\left(H_{j-1}-H_{j}\right)+\alpha_{j+1}\left(H_{j+1}-H_{j}\right) .
$$

Given that $0 \leq T_{j}(x) \leq 1$ by a simple application of the maximum principle, the bounds for $\left(T_{j}\right)_{x}$ are now obtained in the same manner as above.

The bulk burning rate for the model (2.1) is now defined accordingly via

$$
V(t)=\frac{1}{H} \sum_{j=1}^{N} h_{j} \int_{-\infty}^{\infty}\left(T_{j}\right)_{t}(x) d x .
$$

Assuming the initial data satisfies (2.2), the indefinite integrals are well-defined (integrating over $[-B, B]$ and taking $B$ to infinity). As a consequence of (2.1), Lemma 2.1, and the condition $\sum_{j=1}^{N} h_{j} A_{j}=0$, we also have

$$
V(t)=\frac{1}{H} \sum_{j=1}^{N} h_{j} \int_{-\infty}^{\infty} T_{j}(x)\left(1-T_{j}(x)\right) d x .
$$

We continue by computing:

$$
\begin{array}{r}
V^{\prime}(t)=\partial_{t} \frac{1}{H} \sum_{j=1}^{N} h_{j} \int_{-\infty}^{\infty} T_{j}(x)\left(1-T_{j}(x)\right) d x=\frac{1}{H} \sum_{j=1}^{N} h_{j} \int_{-\infty}^{\infty}\left(T_{j}\right)_{t}(x)\left(1-2 T_{j}(x)\right) d x \\
=\frac{1}{H} \sum_{j=1}^{N} h_{j} \int_{-\infty}^{\infty}\left(2\left(\left(T_{j}\right)_{x}\right)^{2}+T_{j}\left(1-T_{j}\right)\left(1-2 T_{j}(x)\right)+\frac{2 \kappa}{h_{j}}\left(T_{j}(x)-T_{j-1}(x)\right)^{2}\right) d x \\
\geq \frac{1}{H} \sum_{j=1}^{N} h_{j} \int_{-\infty}^{\infty}\left(2\left(\left(T_{j}\right)_{x}\right)^{2}+\frac{2 \kappa}{h_{j}}\left(T_{j}(x)-T_{j-1}(x)\right)^{2}\right) d x-V(t) .
\end{array}
$$

In the second step above, we substitute $\left(T_{j}\right)_{t}(x)$ from $(2.1)$, integrate by parts, and rearrange the terms. These manipulations are justified by Lemma 2.1. In the last step we use the expression (2.8) and the fact that $0 \leq T_{j}(x) \leq 1$.

As a warm up before our main results, we prove the following estimate.

TheOREM 2.2. For any choice of $A_{j}$ such that $\sum_{j} h_{j} A_{j}=0$, we have

$$
V(t)^{2} \geq \frac{\pi^{2}}{16}+e^{-2 t}\left(V(0)^{2}-\frac{\pi^{2}}{16}\right) .
$$


A key element in the proof is a general functional inequality.

Proposition 2.3. For any function $T(x) \in C^{1}(\mathbb{R}), 0 \leq T(x) \leq 1$, such that $T(x) \rightarrow 1$ as $x \rightarrow-\infty$ and $T(x) \rightarrow 0$ as $x \rightarrow \infty$, we have

$$
\int_{-\infty}^{\infty}\left(T_{x}\right)^{2} d x \int_{-\infty}^{\infty} T(1-T) d x \geq\left(\frac{\pi}{8}\right)^{2}
$$

The constant $\left(\frac{\pi}{8}\right)^{2}$ is sharp.

Proof. We note that the inequality (2.11) has been proved in [9] with a weaker universal constant on the right hand side. Using the Cauchy-Schwartz inequality we find that

$$
\int_{-\infty}^{\infty}\left(T_{x}\right)^{2} d x \int_{-\infty}^{\infty} T(1-T) d x \geq\left(\int_{-\infty}^{\infty} T_{x}(T(1-T))^{1 / 2} d x\right)^{2} .
$$

Changing the variable and taking into account the asymptotic behavior of $T(x)$, we have

$$
\int_{-\infty}^{\infty} T_{x}(T(x)(1-T(x)))^{1 / 2} d x=\int_{0}^{1}(T(1-T))^{1 / 2} d T .
$$

The latter integral is computed explicitly by setting $T=\cos \theta$, and evaluates to $\frac{\pi}{8}$. This proves (2.11). To show that this constant is sharp, note that if $T_{x}=(T(1-T))^{1 / 2}$, then

$$
\int_{-\infty}^{\infty}\left(T_{x}\right)^{2} d x \int_{-\infty}^{\infty} T(1-T) d x=\left(\int_{-\infty}^{\infty} T_{x}(T(1-T))^{1 / 2} d x\right)^{2}=\left(\frac{\pi}{8}\right)^{2}
$$

We can solve for $T(x)$ explicitly and use the solution to construct an explicit function satisfying the necessary asymptotic behavior for which the equality in (2.11) is achieved. In particular,

$$
T(x)= \begin{cases}1 & \text { if } x \leq-\frac{\pi}{2} \\ \frac{1-\sin x}{2} & \text { if }-\frac{\pi}{2} \leq x \leq \frac{\pi}{2} \\ 0 & \text { if } \frac{\pi}{2} \leq x\end{cases}
$$

is one such function.

Proof. [Proof of Theorem 2.2] From (2.9), we see that

$$
V^{\prime}(t)+V(t) \geq \frac{2}{H} \sum_{j=1}^{N} h_{j} \int_{-\infty}^{\infty}\left(\left(T_{j}\right)_{x}\right)^{2} d x .
$$


From (2.2) and Proposition 2.3, we derive

$$
\begin{array}{r}
V^{\prime}(t)+V(t) \geq \frac{\pi^{2}}{32 H} \sum_{j=1}^{N} \frac{h_{j}}{\int_{-\infty}^{\infty} T_{j}(x)\left(1-T_{j}(x)\right) d x} \\
\geq \frac{\pi^{2} H}{32}\left(\sum_{j=1}^{N} h_{j} \int_{-\infty}^{\infty} T_{j}(x)\left(1-T_{j}(x)\right) d x\right)^{-1}=\frac{\pi^{2}}{32 V(t)} .
\end{array}
$$

On the second step we use Cauchy-Schwartz inequality. Therefore, we have

$$
\frac{d}{d t}\left(V(t)^{2}\right)+2 V(t)^{2} \geq \frac{\pi^{2}}{16}
$$

The inequality (2.10) follows from the standard application of the Gronwall lemma.

Theorem 2.2 gives a universal lower bound on the reaction rate, independent of the flow. One consequence is that in the framework of our model, a mean zero flow cannot quench the reaction. However, we are interested in the bounds that show enhancement of the reaction. We will prove such bounds in the next two sections.

\section{The two-layer model}

For the sake of clarity, we start with a simplified version of our general model (2.1) where only two layers of unit width are involved. Namely, we look at a system

$$
\begin{aligned}
& \left(T_{1}\right)_{t}+A\left(T_{1}\right)_{x}-\left(T_{1}\right)_{x x}=T_{1}\left(1-T_{1}\right)+\kappa\left(T_{2}-T_{1}\right) \\
& \left(T_{2}\right)_{t}-A\left(T_{2}\right)_{x}-\left(T_{2}\right)_{x x}=T_{2}\left(1-T_{2}\right)+\kappa\left(T_{1}-T_{2}\right)
\end{aligned}
$$

with the initial data $T_{1,2}(x, 0)$ satisfying $(2.2)$. We set $h_{1}=h_{2}=1$ in the definition (2.7) of the bulk burning rate. Throughout the rest of the paper we denote by $C$ different universal constants which enter the estimates.

THEOREM 3.1. There exists a universal positive constant $C$ such that for any $\tau>0$, we have

$$
\frac{1}{\tau} \int_{0}^{\tau} V(t) d t \geq C A\left[\frac{1}{\kappa \tau^{2}}+\frac{1}{\kappa \tau}+\frac{1}{\tau}+\kappa+1\right]^{-1}
$$

REMARK 3.2. Note that Theorem 3.1 is a particular case of Theorem 1.1 for $N=2$.

Proof. We first integrate (3.1) over the entile real line. Given the asymptotic behavior of $T_{1}$ and $T_{2},(2.2)$ and Lemma 2.1, we obtain

$$
\begin{aligned}
& \int_{\mathbb{R}}\left(T_{1}\right)_{t} d x-A=\int_{\mathbb{R}} T_{1}\left(1-T_{1}\right) d x+\kappa \int_{\mathbb{R}}\left(T_{2}-T_{1}\right) d x \\
& \int_{\mathbb{R}}\left(T_{2}\right)_{t} d x+A=\int_{\mathbb{R}} T_{2}\left(1-T_{2}\right) d x+\kappa \int_{\mathbb{R}}\left(T_{1}-T_{2}\right) d x
\end{aligned}
$$


Subtracting (3.4) from (3.3) and integrating the result over $t \in\left[\tau_{1}, \tau_{2}\right]$, we find that

$$
\begin{aligned}
& \int_{\mathbb{R}}\left(T_{1}\left(\tau_{2}\right)-T_{2}\left(\tau_{2}\right)\right) d x-\int_{\mathbb{R}}\left(T_{1}\left(\tau_{1}\right)-T_{2}\left(\tau_{1}\right)\right) d x \\
& +\int_{\tau_{1}}^{\tau_{2}} \int_{\mathbb{R}}\left(T_{2}\left(1-T_{2}\right)-T_{1}\left(1-T_{1}\right)\right) d x d t \\
& +2 \kappa \int_{\tau_{1}}^{\tau_{2}} \int_{\mathbb{R}}\left(T_{1}-T_{2}\right) d x d t=2 A\left(\tau_{2}-\tau_{1}\right) .
\end{aligned}
$$

We now claim the following Lemma.

Lemma 3.3. For any $t>0$, we have

$$
\begin{gathered}
\int_{\mathbb{R}}\left|T_{1}(x, t)-T_{2}(x, t)\right| d x \leq 3\left[\int_{\mathbb{R}}\left(T_{1}(x, t)-T_{2}(x, t)\right)^{2} d x+\right. \\
\left.\int_{\mathbb{R}}\left(T_{1}(x, t)\left(1-T_{1}(x, t)\right)+T_{2}(x, t)\left(1-T_{2}(x, t)\right)\right) d x\right] \leq \frac{3}{\kappa}\left(V(t)+V^{\prime}(t)\right)+3 V(t) .
\end{gathered}
$$

Proof. We begin by proving the first inequality, which is valid for any functions $T_{1}(x), T_{2}(x) \in L^{1}(\mathbb{R}) \cap L^{2}(\mathbb{R})$ :

$$
\int_{\mathbb{R}}\left|T_{1}-T_{2}\right| d x \leq 3\left[\int_{\mathbb{R}}\left(T_{1}-T_{2}\right)^{2} d x+\int_{\mathbb{R}}\left(T_{1}\left(1-T_{1}\right)+T_{2}\left(1-T_{2}\right)\right) d x\right] .
$$

Consider two different cases. Denote $S$ the set of all $x$ such that $\left|T_{1}(x)-T_{2}(x)\right| \geq 1 / 3$. Then $\left|T_{1}(x)-T_{2}(x)\right| \leq 3\left|T_{1}(x)-T_{2}(x)\right|^{2}$ and (3.7) hold if we restrict the integration to set $S$. Next, assume that $\left|T_{1}(x)-T_{2}(x)\right| \leq 1 / 3$. Note that if at least one of $T_{1}(x)$, $T_{2}(x)$ belongs to the interval $[1 / 3,2 / 3]$, then $T_{1}(x)\left(1-T_{1}(x)\right)+T_{2}(x)\left(1-T_{2}(x)\right) \geq 2 / 9$. Consider the alternative where both $T_{1}(x)$ and $T_{2}(x)$ lie in either $[0,1 / 3)$ or $(2 / 3,1]$. Note that the absolute value of the derivative of function $x(1-x)$ satisfies $|(1-2 x)| \geq$ $1 / 3$ in these intervals. Then by the mean value theorem,

$$
\begin{aligned}
T_{1}(x)\left(1-T_{1}(x)\right)+T_{2}(x)\left(1-T_{2}(x)\right) & \geq \mid T_{1}(x)\left(1-T_{1}(x)\right) \\
-T_{2}(x)\left(1-T_{2}(x)\right) \mid & \geq \frac{1}{3}\left|T_{1}(x)-T_{2}(x)\right| .
\end{aligned}
$$

Therefore, if $x \in \mathbb{R} \backslash S$, then

$$
\left|T_{1}(x)-T_{2}(x)\right| \leq 3\left(T_{1}(x)\left(1-T_{1}(x)\right)+T_{2}(x)\left(1-T_{2}(x)\right)\right) .
$$

Combining the two cases, we obtain (3.7).

Now the second inequality in (3.6) follows from the definition (2.7) of bulk burning rate, $V(t)$, and the estimate (2.9) (recall that we set $h_{1,2}=1$ ).

Given Lemma 3.3, (3.5) implies that

$$
\frac{3}{\kappa}\left[V^{\prime}\left(\tau_{2}\right)+V^{\prime}\left(\tau_{1}\right)\right]+\left(\frac{3}{\kappa}+9\right)\left[V\left(\tau_{2}\right)+V\left(\tau_{1}\right)\right]+(6 \kappa+7) \int_{\tau 1}^{\tau 2} V(t) d t \geq 2 A\left(\tau_{2}-\tau_{1}\right) .
$$


Let us now apply the following triple averaging in time over $t \in[0, \tau]$ to the difference of equations (3.3) and (3.4):

$$
\frac{1}{\tau^{3}} \int_{0}^{\frac{\tau}{4}} \int_{\frac{\tau}{4}-s}^{\frac{\tau}{4}+s} \int_{\frac{\tau}{2}-k}^{\frac{\tau}{2}+k} \cdot d t d k d s=\frac{1}{\tau^{3}} \int_{0}^{\tau} \cdot G\left(\frac{\tau}{2}, t-\frac{\tau}{2}\right) d t
$$

where

$$
G(h, \xi)= \begin{cases}\frac{1}{2}(h-|\xi|)^{2}-\left(\frac{h}{2}-|\xi|\right)^{2} & \text { if } 0 \leq|\xi| \leq \frac{h}{2} \\ \frac{1}{2}(h-|\xi|)^{2} & \text { if } \frac{h}{2} \leq|\xi| \leq h .\end{cases}
$$

The formula (3.9) can be checked by routine change in the order of integration. After the first integration, we obtain (3.5) and thus (3.8) with $\tau_{1}=\tau-k, \tau_{2}=\tau+k$. Integrating two more times and changing the order of integration leads to the following estimate:

$$
\begin{array}{r}
\frac{3}{\kappa} \int_{0}^{\tau} V(t) d t+\left(\frac{3}{\kappa}+9\right) \int_{0}^{\tau} V(t) h(t, \tau) d t+ \\
(6 \kappa+7) \int_{0}^{\tau} V(t) G\left(\frac{\tau}{2}, t-\frac{\tau}{2}\right) d t \geq \frac{A \tau^{3}}{16}
\end{array}
$$

where

$$
h(t, \tau)= \begin{cases}t \quad \text { if } 0 \leq t \leq \frac{\tau}{4} \\ \frac{\tau}{4} \quad \text { if } \frac{\tau}{4} \leq t \leq \frac{3 \tau}{4} \\ \tau-t & \text { if } \frac{3 \tau}{4} \leq t \leq \tau\end{cases}
$$

Observe that $G\left(\frac{\tau}{2}, t-\frac{\tau}{2}\right) \leq \frac{\tau^{2}}{8}$ and that $h(t, \tau) \leq \frac{\tau}{4}$. Hence (3.11) can be expressed as follows:

$$
\frac{1}{\tau} \int_{0}^{\tau} V(t) d t \geq C A\left[\frac{1}{\kappa \tau^{2}}+\frac{1}{\kappa \tau}+\frac{1}{\tau}+\kappa+1\right]^{-1}
$$

This coincides with (3.2), thus proving the theorem. It is also easy to estimate that the constant $C$ can be taken equal to $1 / 48$.

We remark that it is easy to accommodate the case where the drift coefficient is time dependent. The same steps as above lead to

$$
\frac{1}{\tau} \int_{0}^{\tau} V(t) d t \geq \frac{C}{\tau^{3}}\left[\frac{1}{\kappa \tau^{2}}+\frac{1}{\kappa \tau}+\frac{1}{\tau}+\kappa+1\right]^{-1} \int_{0}^{\tau} A(t) G\left(\frac{\tau}{2}, t-\frac{\tau}{2}\right) d t .
$$

The consequence of this estimate is that applying averaging time $\tau$ that is smaller than the typical time scale $\tau_{A}$ of $A(t)$ will lead to weaker lower bound in (3.12) due to cancelations in the integral. In particular, if $A(t)$ is periodic, fast varying and mean zero, it is clear that the best estimate one can obtain from (3.12) for the average of the bulk burning rate $V(t)$ will deteriorate as $\tau_{A} \rightarrow 0$. For example, if $A(t)=A \sin \left(t / \tau_{A}\right)$ and $\kappa \sim 1$, then for small $\tau_{A}$ we get scaling $C A \tau_{A}$ for the lower bound on the averages of $V(t)$ over time scales $\tau \sim 1$. This observation is in good agreement with some earlier results obtained in a different model [9]. 


\section{The multi-layer model}

Here we prove a more general version of Theorem 1.1. Let us introduce the notation

$$
R_{1}=1+\max _{j} h_{j}^{-1}, R_{2}=1+\max _{j}\left(h_{j} h_{j-1}^{-1}\right) .
$$

Theorem 4.1. Assume that the initial data $T_{j}(x, 0)$ for (2.1) are front like (2.2). Then there exists a universal constant $C$ such that for any $\tau>0$, we have

$$
\frac{1}{\tau} \int_{0}^{\tau} V(t) d t \geq \frac{C}{H R_{2}} \sum_{j=1}^{N}\left|A_{j-1}-A_{j}\right| h_{j}\left[\frac{1}{\kappa \tau^{2}}+\frac{1}{\kappa \tau}+\frac{R_{1}}{\tau}+\kappa R_{1}+1\right]^{-1} .
$$

REMARK 4.2. Theorem 1.1 is a particular case of Theorem 4.1 when $h_{j} \equiv 1$ (with $\alpha$ substituted by $\kappa)$.

Proof. Consider two neighboring layers, $j$ and $j-1$. Assume without loss of generality that $A_{j} \geq A_{j-1}$. Subtracting the equation for $T_{j}$ from the equation for $T_{j-1}$ and integrating, we find:

$$
\begin{gathered}
\left.\int_{\mathbb{R}}\left(\left(T_{j-1}\right)_{t}-\left(T_{j}\right)_{t}\right) d x+A_{j}-A_{j-1}=\int_{\mathbb{R}}\left(T_{j-1}\left(1-T_{j-1}\right)-T_{j}\left(1-T_{j}\right)\right) d x+4.2\right) \\
\left(\frac{\kappa}{h_{j}}+\frac{\kappa}{h_{j-1}}\right) \int_{\mathbb{R}}\left(T_{j}-T_{j-1}\right) d x+\frac{\kappa}{h_{j}} \int_{\mathbb{R}}\left(T_{j}-T_{j+1}\right) d x+\frac{\kappa}{h_{j-1}} \int_{\mathbb{R}}\left(T_{j-2}-T_{j-1}\right) d x .
\end{gathered}
$$

Multiplying (4.2) by $h_{j}$, and summing over $j=1, \ldots, N$, we obtain:

$$
\begin{gathered}
\sum_{j=1}^{N}(-1)^{\sigma_{j}} h_{j} \int_{\mathbb{R}}\left(T_{j-1}-T_{j}\right)_{t} d x+\sum_{j=1}^{N} h_{j}\left|A_{j-1}-A_{j}\right|= \\
-\sum_{j=1}^{N}(-1)^{\sigma_{j}} h_{j} \int_{\mathbb{R}} T_{j}\left(1-T_{j}\right) d x+\sum_{j=1}^{N}(-1)^{\sigma_{j}} h_{j-1} \int_{\mathbb{R}} T_{j-1}\left(1-T_{j-1}\right) d x \frac{h_{j}}{h_{j-1}} \\
+\sum_{j=1}^{N}(-1)^{\sigma_{j}} \kappa\left[\left(1+\frac{h_{j}}{h_{j-1}}\right) \int_{\mathbb{R}}\left(T_{j}-T_{j-1}\right) d x+\int_{\mathbb{R}}\left(T_{j}-T_{j+1}\right) d x\right. \\
\left.+\int_{\mathbb{R}}\left(T_{j-2}-T_{j-1}\right) d x \frac{h_{j}}{h_{j-1}}\right],
\end{gathered}
$$

where $\sigma_{j}=0$ if $A_{j} \geq A_{j-1}$ and $\sigma_{j}=1$ otherwise. Denote the last sum in (4.3) by $I_{1}$. Using Lemma 3.3 and (2.9), we can estimate:

$$
\begin{gathered}
\left|I_{1}\right| \leq C \kappa \sum_{j=1}^{N}\left[\int_{\mathbb{R}}\left(T_{j}-T_{j-1}\right)^{2} d x+\int_{\mathbb{R}} T_{j}\left(1-T_{j}\right)\right]\left(1+\max _{j}\left(\frac{h_{j}}{h_{j-1}}\right)\right) \\
\leq C H\left(R_{2} V^{\prime}(t)+\kappa V(t) R_{1} R_{2}\right) .
\end{gathered}
$$

As in the proof of Theorem 3.1, we average in $t \in[0, \tau]$ according to (3.9) and apply Lemma 3.3 to estimate the expressions arising from the time derivatives in (4.3) in terms of $V$ and $V^{\prime}$. Taking into account (4.4), after the first integration we obtain, 
with $\tau_{1}=\frac{\tau}{2}-k$ and $\tau_{2}=\frac{\tau}{2}+k$,

$$
\begin{array}{r}
\sum_{j=1}^{N}(-1)^{\sigma_{j}} h_{j}\left[\int_{\mathbb{R}}\left(T_{j-1}\left(\tau_{2}\right)-T_{j}\left(\tau_{2}\right)\right) d x-\int_{\mathbb{R}}\left(T_{j-1}\left(\tau_{1}\right)-T_{j}\left(\tau_{1}\right)\right) d x\right]+ \\
\left(\tau_{2}-\tau_{1}\right) \sum_{j=1}^{N} h_{j}\left|A_{j-1}-A_{j}\right| \leq C H R_{2}\left(V\left(\tau_{2}\right)-V\left(\tau_{1}\right)+\left(\kappa R_{1}+1\right) \int_{\tau_{1}}^{\tau_{2}} V(t) d t\right) .
\end{array}
$$

Applying Lemma 3.3 once again to the expression on the left hand side, and integrating twice more, we obtain

$$
\begin{array}{r}
\frac{\tau^{3}}{H} \sum_{j=1}^{N} h_{j}\left|A_{j-1}-A_{j}\right| \leq C R_{2}\left[\left(\kappa R_{1}+1\right) \int_{0}^{\tau} V(t) G\left(\frac{\tau}{2}, t-\frac{\tau}{2}\right) d t+\right. \\
\left.\left(\kappa^{-1}+R_{1}\right) \int_{0}^{\tau} V(t) h(t, \tau) d t+\kappa^{-1} \int_{0}^{\tau} V(t) d t\right] .
\end{array}
$$

Taking into account the bounds on functions $G$ and $h$, we arrive at

$$
\frac{1}{\tau} \int_{0}^{\tau} V(t) d t \geq \frac{C}{H R_{2}} \sum_{j=1}^{N} h_{j}\left|A_{j-1}-A_{j}\right|\left(1+\kappa R_{1}+\frac{1}{\kappa \tau^{2}}+\frac{1}{\kappa \tau}+\frac{R_{1}}{\tau}\right)^{-1} .
$$

This completes the proof of Theorem 4.1. In the situation where all $h_{j} \equiv 1$, and hence $R_{1}=R_{2}=2$, we obtain Theorem 1.1 exactly. In general, we see that small $h_{j}$ (which translate to the large coupling constants $\alpha_{j}$ ), or strongly non-uniform size distributions of the neighboring $h_{j}$, weaken the lower bound.

\section{Numerical simulations}

We carried out numerical simulations for the two layer system (3.1). Our main interest was in finding the reaction propagation rate in the established regime, depending on $A$ and $\kappa$. Our results are obtained using the Crank-Nicholson implicit scheme. The implementation features two noteworthy modifications. Firstly, the transient wave that occurs between the initial condition and the eventual steady wavefront shape was ignored when calculating the front speed. This was achieved by using a "transient" parameter, and then ignoring the wave speed for times 0 to $t_{\text {transient }}$. Secondly, we employed a computational trick to expedite simulation. In a naive implementation, we would apply the Crank-Nicholson scheme to the entire field of points that the wave travels through. Since the matrices involved in the computation are $n \times n$ where $n$ is the number of points being considered, considering the entire field of points that the wave passes through can be computationally expensive. We avoided this by only applying Crank-Nicholson to points in the immediate neighborhood of the front itself. Then after every iteration we adjusted our calculation frame to stay centered on the wavefront, making sure that the value of the temperature at the border of the computational domain remains close to 0 and 1 respectively.

We found clear linear dependence on the flow strength parameter $A$. The Figure 5.1 shows this linear dependence for different values of the coupling constant $\kappa$. The constant $\kappa$ varies from 0.1 to 1 with 0.1 step. The slope of the graph is monotone decreasing in $\kappa$, so that the steeper slopes on Figure 5.1 correspond to smaller values 


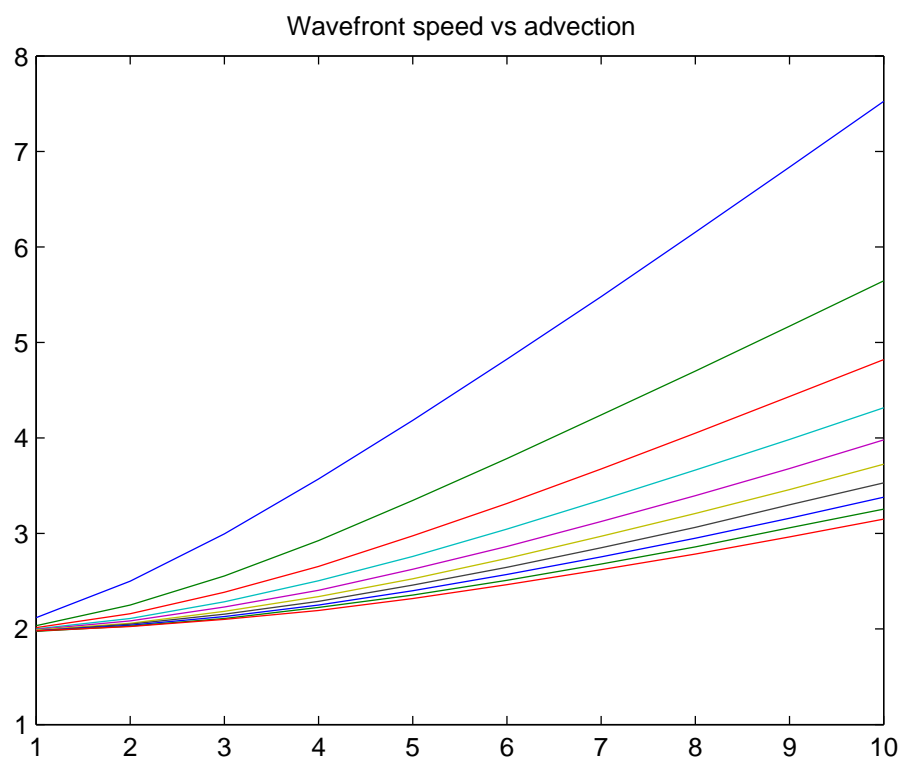

FIG. 5.1. The bulk burning rate versus flow strength $A$ for different values of the coupling $\kappa$

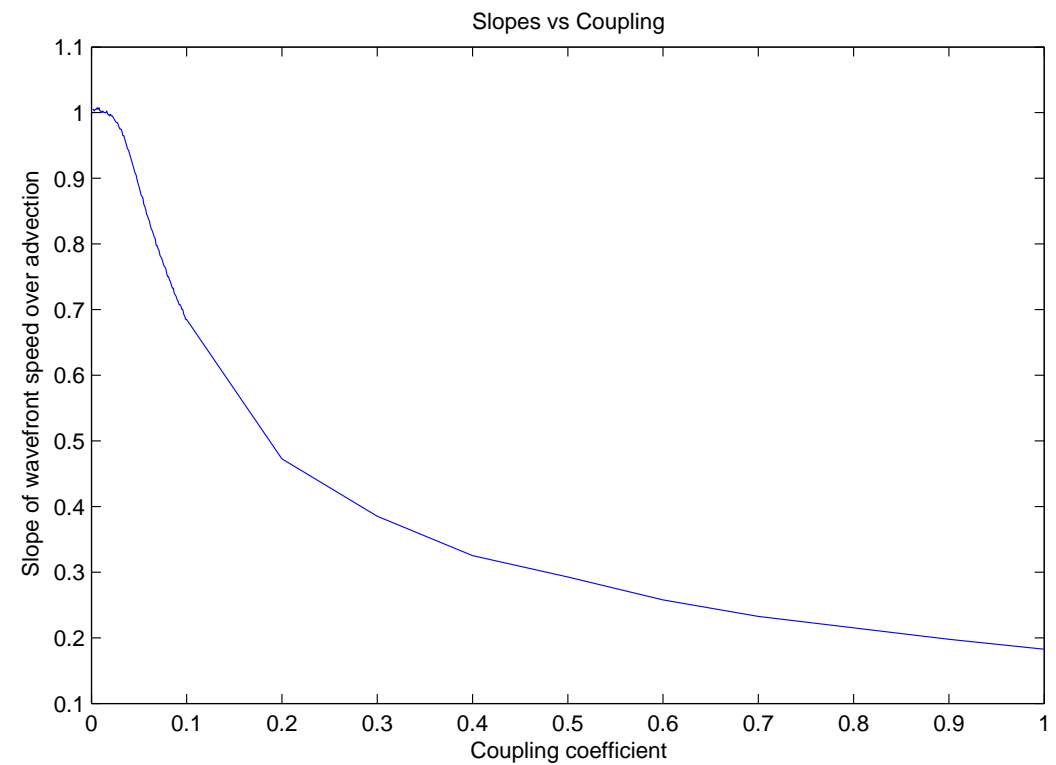

FIG. 5.2. The slopes corresponding to different values of $\kappa$

of $\kappa$. The intuitive explanation for this effect is that stronger diffusion between layers mollifies the front stretching produced by the flow, thus reducing the reaction zone. 
The graph on Figure 5.2 shows the dependence of slope on $\kappa$. The shape of the graph is in good qualitative agreement with the bound of Theorem 3.1. Notice that the slope tends to one as $\kappa \rightarrow 0$. A simple intuitive explanation of this effect is that $T_{1}$ satisfies

$$
\left(T_{1}\right)_{t}+A\left(T_{1}\right)_{x}-\left(T_{1}\right)_{x x} \geq T_{1}\left(1-T_{1}\right)-\kappa T_{1} .
$$

Thus one may expect that as $\kappa \rightarrow 0$, the solution will propagate forward at least as fast as the usual KPP traveling wave shifted forward with speed $A$. On the other hand, a supersolution argument similar to one employed in [9] can be used to show that the propagation speed cannot exceed $A+2$ (where 2 is the lowest KPP speed in our normalization).

We note that the results of our simulations are similar to the results of [30], where combustion in a shear flow was studied in a full PDE setting by means of a more sophisticated numerical scheme.

Acknowledgments. This work has been a part of Collaborative Undergraduate Research Lab (CURL) at the University of Wisconsin during the 2004-2005 academic year. Support of the NSF VIGRE grant is gratefully acknowledged. We thank Carl Edquist, Morgan Franklin, Jeremy Jacobson, Paul Heideman, Julie Mitchell and John Vano for interesting discussions and help with this project. We are especially indebted to Paul Milewski since without his assistance with the code, the numerical simulations would be still running. The work of LRM, DA and EB was supported in part by the NSF VIGRE grant. The work of AK has been partially supported by the Alfred P. Sloan Research Fellowship and NSF-DMS grant 0314129 .

\section{REFERENCES}

[1] M. Abel, A. Celani, D. Vergni and A. Vulpiani, Front propagation in laminar flows, Phy. Rev. E, 64, 6307, 2001.

[2] M. Abel, M. Cencini, D. Vergni and A. Vulpiani, Front speed enhancement in cellular flows, Chaos, 12(2), 481-488, 2002

[3] D. Aronson and H. Weinberger, Multidimensional nonlinear diffusion arising in population genetics, Adv. in Math., 30, 33-76, 1978.

[4] B. Audoly, H. Berestycki and Y. Pomeau, Réaction diffusion en écoulement stationnaire rapide, C. R. Acad. Sci., Ser. IIB, 328, 255-262, 2000.

[5] H. Berestycki, The influence of advection on the propagation of fronts in reaction-diffusion equations, Nonlinear PDEs in Condensed Matter and Reactive Flows, NATO Science Series C, H. Berestycki and Y. Pomeau eds, Kluwer, Doordrecht, 569, 2003.

[6] H. Berestycki, B. Larrouturou and J.-M. Roquejoffre, Stability of traveling fronts in a model for flame propagation I: linear stability, Arch. Rat. Mech. Anal., 117, 97-117, 1992.

[7] J. D. Buckmaster and G. S. S Ludford, Theory of Laminar Flames, Cambridge University Press, Cambridge-New York, 1982.

[8] P. Clavin and F. A. Williams, Theory of pre-mixed flame propagation in large-scale turbulence, J. Fluid Mech., 90, 589-604, 1979.

[9] P. Constantin, A. Kiselev, A. Oberman and L. Ryzhik, Bulk burning rate in passive - reactive diffusion, Arch. Rat. Mech. Anal., 154, 53-91, 2000.

[10] P. Constantin, A. Kiselev and L. Ryzhik, Quenching of flames by fluid advection, Comm. Pure Appl. Math., 54, 1320-1342, 2001.

[11] L. C. Evans, Partial Differential Equations, Graduate Studies in Mathematics, AMS, Providence, 1998

[12] R. Fisher, The wave of advance of advantageous genes, Ann. Eugenics, 7, 355-369, 1937.

[13] M. Freidlin, Functional Integration and Partial Differential equations, Ann. Math. Stud., Princeton University Press, Princeton, 109, 1985.

[14] F. Hamel and N. Nadirshvili, Travelling fronts and entire solutions of the Fisher-KPP equation in $R^{N}$, Arch. Rat. Mech. Anal., 157, 91-163, 2001. 
[15] S. Heinze, G. Papanicolau and A. Stevens, Variational principles for propagation speeds in inhomogeneous media, SIAM J. Appl. Math., 62, 129-148, 2001.

[16] L. Kagan and G. Sivashinsky, Flame propagation and extinction in large-scale vortical flows, Combust. Flame, 120, 222-232, 2000.

[17] L. Kagan, P. D. Ronney and G. Sivashinsky, Activation energy effect on flame propagation in large-scale vortical flows, Combust. Theory Modelling, 6, 479-485, 2002.

[18] B. Khouider, A. Bourlioux and A. Majda, Parametrizing the burning speed enhancement by small-scale periodic flows. I. Unsteady shears, flame residence time and bending, Combust. Theory Model, 5, 295-318, 2001.

[19] B. Khouider and A. Bourlioux, Computing the effective Hamiltonian in the Majda-Souganidis model of turbulent premixed flames, SIAM J. Numer. Anal., 40, 1330-1353, 2002.

[20] A. Kiselev and L. Ryzhik, Enhancement of the traveling front speeds in reaction-diffusion equations with advection, Ann. de l'Inst. Henri Poincaré, C. Analyse non linéaire, 18, 309358, 2001.

[21] A. N. Kolmogorov, I. G. Petrovskii and N. S. Piskunov, Étude de l'équation de la chaleur de matière et son application à un problème biologique, Bull. Moskov. Gos. Univ. Mat. Mekh., 1, 1-25 1937. (see [28] pp. 105-130 for an English transl.)

[22] S. Levin, The problem of pattern and scale in ecology, Ecology, 73, 1943-1967, 1992.

[23] A. Majda and P. Souganidis, Large scale front dynamics for turbulent reaction-diffusion equations with separated velocity scales, Nonlinearity, 7, 1-30, 1994.

[24] J. D. Murray, Mathematical Biology, Springer-Verlag, Berlin Heidelberg, 1993.

[25] J. Nolen and J. Xin, A variational principle based study of KPP minimal front speeds in random shears, Nonlinearity, 18, 1655-1675, 2005.

[26] J. Nolen, M. Rudd and J. Xin, Existence of KPP fronts in spatially-temporally periodic advection and variational principle for propagation speeds, Dynamics of PDE, 2, 1-24, 2005.

[27] J. Nolen and J. Xin, Min-max variational principle and front speeds in random shear flows, Methods and Applications of Analysis, 11, 635-644, 2004.

[28] Dynamics of Curved Fronts, P. Pelcé, Ed., Academic Press, 1988.

[29] N. A. Peters, Turbulent Combustion, Cambridge University Press, Cambridge, 2000.

[30] N. Vladimirova, P. Constantin, A. Kiselev, O. Ruchayskiy and L. Ryzhik, Flame enhancement and quenching in fluid flows, Combust. Theory Model, 7, 487-508, 2003.

[31] A. Volpert, V. Volpert and V. Volpert, Traveling Wave Solutions of Parabolic Systems, Translations of mathematical Monographs, Amer. Math. Soc., Providence, Rhode Island, 140, 1994.

[32] F. A. Williams, Combustion Theory, Benjamin-Cummings, Menlo Park, 1985.

[33] J. Xin, Front propagation in heterogeneous media, SIAM Rev., 42, 161-230, 2000.

[34] Ya. B. Zeldovich, G. I. Barenblatt, V. B. Librovich and G. M. Makhviladze, The Mathematical Theory of Combustion and Explosions, Consultants Bureau (Plenum), New York, 1985. 